\title{
Maxillary Sinusitis by Staphylococcus aureus Infection in a Thoroughbred Gelding: Case Report
}

\author{
Sang Kyu Lee \\ Inhyung Lee L $^{2 *}$ \\ 'KRA Veterinary Center, Korea Racing \\ Authority, Gwacheon 13822, Korea \\ ${ }^{2}$ Department of Veterinary Clinical Scienc- \\ es, College of Veterinary Medicine and \\ Research Institute for Veterinary Science, \\ Seoul National University, Seoul 08826, \\ Korea
}

*Correspondence: inhyunglee@snu.ac.kr

\section{ORCID}

Sang Kyu Lee:

https://orcid.org/0000-0002-8117-914X

Inhyung Lee:

https://orcid.org/0000-0002-0712-7165

Copyright $\odot$ The Korean Society of Veterinary Clinics
Abstract A 4-year-old gelding Thoroughbred racehorse, which had been undergoing antibiotic therapy at a local veterinary clinic, was referred to the KRA veterinary center with a 20-day history of continuous right nasal discharge. Patient's history, endoscopic examination, and radiographic examination revealed primary maxillary sinusitis. Under sedation, surgical intervention was performed to collect samples and remove the accumulated mucopurulent exudate in the sinus. Swab samples were collected from the sinus during surgery for cytology and antimicrobial susceptibility testing. Only one type of bacteria was cultured, and molecular analyses of $16 \mathrm{~S}$ ribosomal RNA gene sequences identified it as Staphylococcus aureus (S. aureus). The isolate was resistant to multiple antibiotics, which are frequently used in equine practice. Trimethoprim-sulfamethoxazole was chosen based on antibiotic susceptibility test, trephination, and sinus lavage using saline were applied to treat bacterial sinusitis. The clinical signs improved after 1 month and the patient resumed training. This report describes $S$. aureus isolated from bacterial maxillary sinusitis in a horse and its antibiotic susceptibility.

Key words sinusitis, horse, Staphylococcus aureus, parasinus, antibiotic susceptibility. 


\section{Introduction}

Sinusitis is a relatively rare respiratory disease in horses, and it has been reported that at least 75 out of 17,000 horses presented to veterinary clinics according to an equine practice survey (24). Although sinusitis occurs infrequently, it is a clinically important disease because of difficulties in treatment and the chronic nature of the disease $(18,24)$. Sinusitis occurs mainly in the paranasal sinuses of horses (3), and it is categorized as primary and secondary sinusitis upon its causes $(9,18)$. Primary sinusitis is followed by an upper respiratory tract infection of the paranasal sinuses $(9,18)$. Secondary sinusitis occurs as a consequence of other diseases, commonly by dental disorders involving tooth root infection and less frequently by trauma, sinus cysts, or neoplasm $(8-10,24)$.

Clinical signs of primary sinusitis are nonspecific and it depends on the affected sinus and chronicity of the disease. Nasal discharge from the affected side, which is usually unilateral, is a common clinical sign, and less commonly, facial distortion can be found (9). Streptococcus equi (S. equi) and Streptococcus equi subsp. zooepidemicus (S. zooepidemicus) are the most common bacterial isolates in equine primary sinusitis $(9,21,25)$. Facial distortion, fetid nasal discharge, and draining facial tracts are related to secondary sinusitis (9).

Treatment of primary sinusitis involves sinus lavage with sterile saline and administration of systemic antibiotics $(9,19)$. Antibiotic selection is generally recommended based on the sample culture and sensitivity test results $(9,10)$. While awaiting the test results, penicillin, gentamicin, and trimethoprim-sulfamethoxazole are recommended antibiotics for

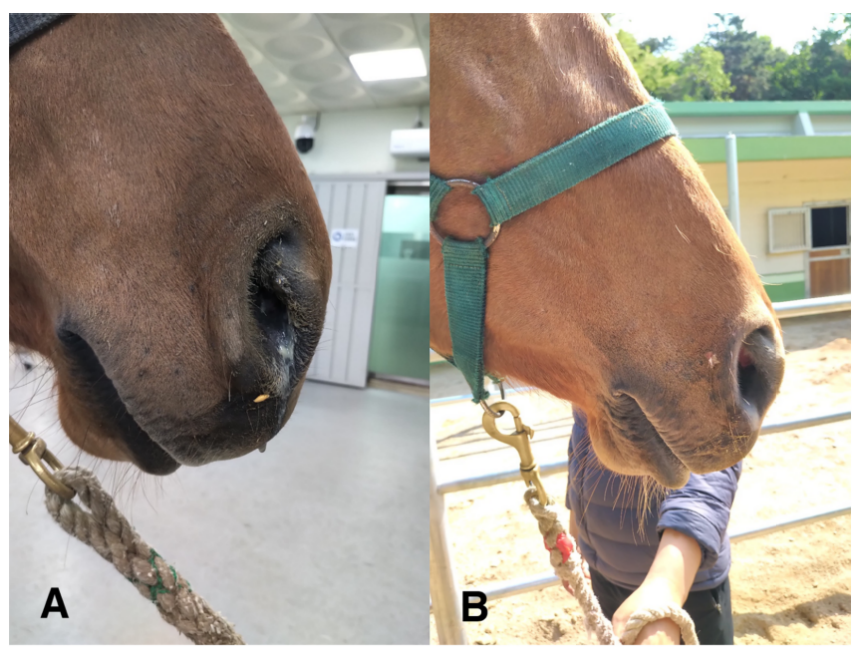

Fig. 1. Unilateral mucopurulent exudate from right nostril at admission (A) and reduced nasal discharge from right nostril on postoperative day 28 (B). primary sinusitis because they are reported to be efficacious against the most common isolates from equine sinusitis $(4,8)$. Treatment of secondary sinusitis requires removal of the underlying condition, together with the therapies required for the treatment of primary sinusitis (9).

The present report describes a case of isolation of Staphylococcus aureus (S. aureus) from primary right sinusitis and its antibiotic susceptibility that was treated with properly selected antibiotic therapy along with trephination. This study provides the first clinical description of a staphylococcal infection from sinusitis in horses living in Korea.

\section{Case Report}

A 4-year-old gelding Thoroughbred racehorse, which had been undergoing systemic antibiotic therapy at a local veterinary clinic, was referred to the KRA veterinary center with a 20-day history of continuous right nasal discharge. The patient had a history of intermittent penicillin and gentamicin administration for 20-days by the local veterinary clinic. Despite the conserved therapy, the condition did not improve. Mucopurulent exudate was present from the unilaterally right nostril on physical examination (Fig. 1A). The ventral and middle nasal meati of the right sinusitis were examined using endoscopy. Routine oral examination was performed with an equine mouth gag (Schoupe equine speculum, Jorgensen Laboratories, Loveland, CO, USA), and no abnormality was found in the right maxillary cheek teeth. A lateral radiograph demonstrated fluid lines in both the rostral and caudal maxillary sinuses (Fig. 2A). Based on the patient's history, clinical signs, and endoscopic and radiographic examinations, the

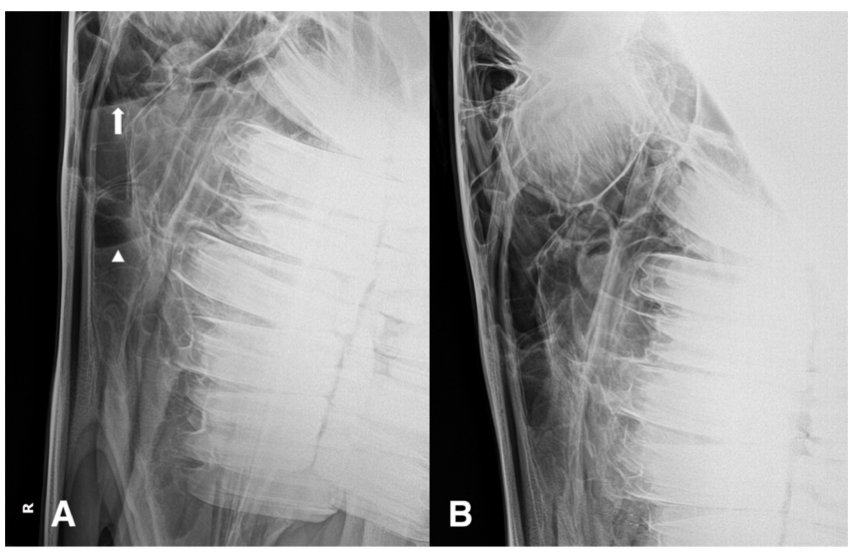

Fig. 2. Lateral radiograph of maxillary sinus. Two fluid lines (indicative of liquid pus) observed in the rostral (arrow head) and caudal maxillary sinuses (white arrow) (A). Fluid lines were absent in lateral radiograph of maxillary sinus on postoperative day 21 (B). 


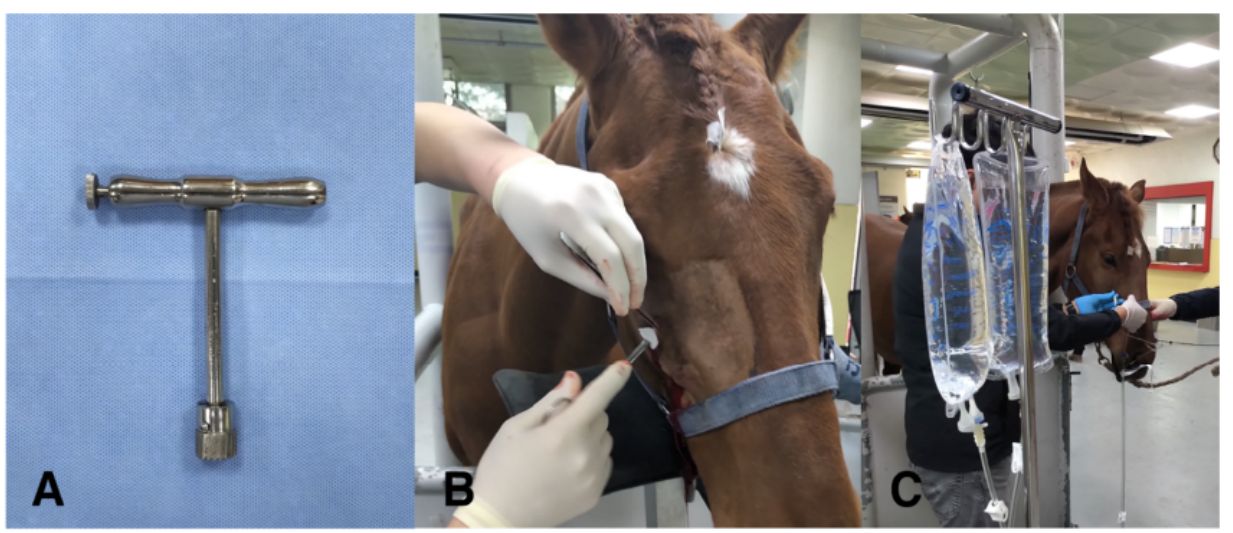

Fig. 3. A 1/2-inch Galt trephine used in trephination (A). Cotton gauze grasped with a forceps was applied into the right maxillary sinus to remove exudate and blood clot after trephination (B). Postoperative sinus lavage being performed using a large volume of warm sterile saline (C). patient was diagnosed with rostral and caudal maxillary sinusitis. Since the patient had a history of antibiotic treatment for over 20-days without improving the condition, surgical intervention was decided to resolve the sinusitis. Trephination of the maxillary sinus was performed as a standing surgery. Before the trephination, intramuscular (IM) gentamicin (6.6 $\mathrm{mg} / \mathrm{kg}$, Daesung gentamicin injection, Daesung microbiological labs, Euiwang, Korea), procaine penicillin G $(22,000 \mathrm{IU} /$ kg IM, PPS injection, Daesung microbiological labs, Euiwang, Korea) and intravenous (IV) meloxicam $(0.5 \mathrm{mg} / \mathrm{kg}$, Meta$\mathrm{cam}^{\circledR}$ solution; Boehringer Ingelheim, Ingelheim, Germany) were administered as a preoperative treatment.

The patient was sedated using detomidine $(0.03 \mathrm{mg} / \mathrm{kg} \mathrm{IV}$, Provet Detomidin ${ }^{\circledR}$, Provet, Istanbul, Turkey). The skin around the right facial crest was clipped and aseptically prepared for trephination with 7.5\% povidone iodine (Poviclean Brush sol., Firson, Cheonan, Korea). The trephine site was determined to be $1 \mathrm{~cm}$ dorsal to the right facial crest and $7 \mathrm{~cm}$ caudal to the rostral end of the facial crest. A total of $5 \mathrm{~mL}$ of $2 \%$ lidocaine (Daihan Lidocaine HCL inj. 2\%, Daihan, Ansan, Korea) was infiltrated subcutaneously using a 25 -gauge needle at the incision site. A $5 \mathrm{~cm}$ long curved incision was made in the skin and the underlying periosteum at the site of trephination. The skin and periosteum were detached from the bone using a curette. The bone was trephined carefully using a Galt trephine (Horsley's Trephine 1/2", Jorgensen Laboratories, Loveland, USA) (Fig. 3A). Dry sterile swabs were placed in the maxillary sinus and rolled over the exudate and sinus mucosa several times. The mucopurulent exudate present in the sinus was drained and removed using cotton gauze (Fig. 3B). The periosteum was sutured with a 2-0 absorbable suture (Ethicon ${ }^{\circledR}$ Vicryl ${ }^{\top \mathrm{M}}$ polyglactin 910 , Somerset County, NJ, USA) in a continuous manner, and the skin was closed with skin staples (Visistat ${ }^{\circledR}$ skin stapler 35R, Teleflex, Morrisville, NC, USA).

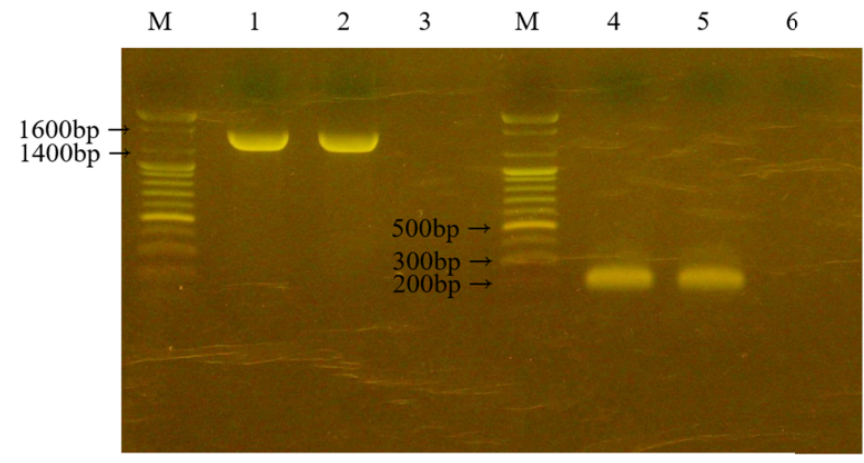

Fig. 4. $P C R$ result of $16 S$ ribosomal RNA gene (Lane 1-3) and icaD gene (Lane 4-6) from the isolated bacterial genomic DNA (S. aureus). M: DNA ladder, 1: sample (1463 bp), 2: positive control, 3: negative control, 4: sample (198 bp), 5: positive control, 6: negative control.

Cytology results revealed neutrophilic inflammation with bacterial infection. Swabs for antimicrobial susceptibility testing were immediately plated on $5 \%$ sheep blood agar plates and incubated for 24 hours at $37^{\circ} \mathrm{C}$. The isolated bacteria were identified molecularly based on the nucleotide sequence of the $16 \mathrm{~S}$ ribosomal RNA gene (Fig. 4) and identified as $S$. aureus (20). The partial nucleotide sequence of the gene was purified using the ExoSAP-IT PCR Product Cleanup (Applied Biosystems, Waltham, MA, USA) and sequenced using a BigDye Terminator v3.1 Cycle Sequencing Kit V.3.1, and analyzed with a 3730xI DNA Analyzer (Applied Biosystems, Waltham, MA, USA) at a commercial institute (Cosmogenetech, Seoul, Korea). The sequence of the isolate was submitted to GenBank (MZ220764). Dilution test for antimicrobial susceptibility (minimal inhibitory concentration (MIC) assay) was performed using a commercial 96-well microdilution well plate (Sensititre ${ }^{\mathrm{TM}}$ Equine EQUIN2F Vet AST Plate, Trek Diagnostic Systems, West Sussex, UK) (15). The isolate was susceptible to 8 out of 16 agents (erythromycin, clarithromycin, cefazolin, oxacillin, enrofloxacin, amikacin, rifampin, 
Table 1. Results of antibiotic susceptibility test by minimal inhibitory concentration (MIC) assay of the isolated Staphylococcus aureus

\begin{tabular}{|c|c|c|c|c|c|c|}
\hline \multirow[b]{2}{*}{ Antibiotics } & \multirow[b]{2}{*}{ Antibiotic class } & \multirow{2}{*}{$\begin{array}{c}\text { MIC values } \\
\text { of } \\
\text { the isolate }\end{array}$} & \multicolumn{3}{|c|}{ MIC Breakpointsb, $\mu \mathrm{g} / \mathrm{mL}$} & \multirow[b]{2}{*}{ Results } \\
\hline & & & Susceptible & $\begin{array}{l}\text { Inter- } \\
\text { mediate }\end{array}$ & Resistant & \\
\hline Erythromycin & Macrolides $^{a}$ & 0.5 & $\leq 0.5$ & $1-4$ & $\geq 8$ & Susceptible \\
\hline Clarithromycin & Macrolides & 0.5 & $\leq 2$ & 4 & $\geq 8$ & Susceptible \\
\hline Chloramphenicol & Phenicols ${ }^{a}$ & $>32$ & $\leq 8$ & 16 & $\geq 32$ & Resistant \\
\hline Cefazolin & $\begin{array}{l}\text { Non-extended spectrum } \\
\text { cephalosporins }\end{array}$ & 2 & $\leq 2$ & 4 & $\geq 8$ & Susceptible \\
\hline Oxacillin & $\begin{array}{l}\text { Anti-staphylococcal } \\
\beta \text {-lactams }\end{array}$ & 2 & $\leq 2$ & - & $\geq 4$ & Susceptible \\
\hline Penicillin & Penicillins & $>8$ & $\leq 0.5$ & 1 & $\geq 2$ & Resistant \\
\hline Enrofloxacin & Fluoroquinolones & 0.12 & $\leq 0.12$ & 0.25 & $\geq 0.5$ & Susceptible \\
\hline Ampicillin & Penicillins & $>16$ & $\leq 0.5$ & 1 & $\geq 2$ & Resistant \\
\hline Minocycline & Tetracyclines $^{a}$ & 0.25 & $\leq 0.12$ & 0.25 & $\geq 0.5$ & Intermediate \\
\hline Doxycycline & Tetracyclines $^{a}$ & 8 & $\leq 0.12$ & $\leq 0.25$ & $\geq 0.5$ & Resistant \\
\hline Tetracycline & Tetracyclines $^{a}$ & $>8$ & $\leq 4$ & 8 & $\geq 16$ & Resistant \\
\hline Amikacin & Aminoglycosides & 4 & $\leq 4$ & 8 & $\geq 16$ & Susceptible \\
\hline Ceftiofur & $\begin{array}{l}\text { Extended-spectrum } \\
\text { cephalosporins }\end{array}$ & $>8$ & $\leq 2$ & 4 & $\geq 8$ & Resistant \\
\hline Gentamicin & Aminoglycosides $^{a}$ & $>8$ & $\leq 4$ & 8 & $\geq 16$ & Resistant \\
\hline Rifampin & Ansamycins $^{a}$ & $<1$ & $\leq 1$ & 2 & $\geq 4$ & Susceptible \\
\hline $\begin{array}{l}\text { Trimethoprim- } \\
\text { sulfamethoxazole }\end{array}$ & Folate pathway inhibitors ${ }^{a}$ & $<0.5 / 9.5$ & $\leq 2 / 38$ & - & $\geq 4 / 76$ & Susceptible \\
\hline
\end{tabular}

${ }^{a}$ Class for S. aureus MDR proposed by Magiorakos et al. in 2012 (16), and MDR was defined as the isolate being non-susceptible to at least one agent in $\geq 3$ antimicrobial classes.

${ }^{\mathrm{b}} \mathrm{MIC}$ breakpoints of the Clinical and Laboratory Standards Institute guideline VET01S were used (15).

trimethoprim-sulfamethoxazole) (Table 1). Multi-drug resistance is regarded as resistant to three or more antimicrobial classes, as proposed by Magiorakos et al. (16). S. aureus was not sensitive to the three antimicrobial classes (Table 1). Biofilm production by isolated $S$. aureus was evaluated by polymerase chain reaction (PCR) test. The presence of the intercellular adhesion (ica) operon was detected by polymerase chain reaction (PCR) targeting icaD gene sequences (Fig. 4), as described previously (1). After the amplification of icaD, the amplicon was shipped for DNA nucleotide sequence analysis to a commercial institute (Cosmogenetech, Seoul, Korea) to confirm the accuracy of the reaction. The sequence homology of the deduced nucleotide sequences to the icaD gene sequence was analyzed using the BLAST search program (National Center for Biotechnology Information). The sequence was compared with previously submitted icaD gene sequences using BLAST and showed 100\% identity and submitted to GenBank (MZ220763).

The patient was prescribed meloxicam $(0.6 \mathrm{mg} / \mathrm{kg}$ IV q24h, Metacam ${ }^{\circledast}$ solution; Boehringer Ingelheim, Ingelheim, Germany) for 3 days, and trimethoprim-sulfamethoxazole
(20 mg/kg PO q12h, Septrin Tab, Samil, Ansan, Korea) for 10 days with a probiotic (50 g PO q12h, Clostridium butyricum (C. butyricum) more than $1.0 \times 10^{7} \mathrm{CFU} / \mathrm{g}$, Handong masarang, Handong, Seoul, Korea) as an adjunctive treatment. A $5 \mathrm{~mm}$ stab skin incision was made at the trephined site for lavage. The sinus was lavaged with warm saline through a 14-gauge catheter while infusion of IV fluid was set three times weekly for a month (Fig. 3C). From postoperative day 7, nasal discharge from the right nostril and mucopurulent exudate in lavaged saline decreased, and it was markedly decreased at the 3-week follow-up evaluation. On postoperative day 14, the incisional site was healed without inflammation, and the skin staples were removed. The fluid lines in the right sinuses were not observed on radiographic examination on postoperative day 21 (Fig. 2B). On postoperative day 28, reduced nasal discharge was observed (Fig. 1B), and the horse resumed training on track.

\section{Discussion}

This study describes the treatment of delayed primary 
maxillary sinusitis infected with $S$. aureus in a Thoroughbred horse. S. equi and S. zooepidemicus are the most common bacterial sinusitis pathogens $(9,21,25)$. Penicillin is commonly used for the treatment of streptococcal infection in horses (23). S. equi has been constantly susceptible to penicillin (23) and $S$. zooepidemicus has been sensitive to penicillin without the occurrence of resistance over three decades (17). In Korea, S. zooepidemicus isolated from horses is sensitive to gentamicin and beta-lactam antibiotics such as penicillin, ampicillin, ceftiofur, oxacillin, and cefoxitin (6). In equine practice, primary sinusitis frequently resolves well with empirical antimicrobials $(3,25)$. The local clinic used penicillin and gentamicin for the treatment of sinusitis in patients without antibiotic sensitivity testing. However, bacterial culture and antibiotic sensitivity testing are strongly recommended for the treatment of bacterial sinusitis (10). After referral, bacterial culture tests revealed that $S$. aureus and its antibiotic sensitivity characteristics were different from those of $S$. equi and zooepidemicus. Therefore, it seems that sinusitis was delayed without improvement of clinical signs with penicillin and gentamicin administration. Medical therapy with antimicrobials alone is not likely to be successful in the condition of fluid accumulation within the sinus (7). Additional trephination, lavage, surgical debridement, and drainage through a bone flap are indicated (7). Horizontal fluid lines within the maxillary sinus were observed on lateral radiographs (Fig. 2A), and trephination was performed for both diagnostic and therapeutic purposes. S. aureus was isolated by culture test, and trimethoprim-sulfamethoxazole was used based on an antibiotic sensitivity test. The patient had been treated with penicillin and gentamicin and only $S$. aureus was isolated from the trephined sinus swabs. The isolated $S$. aureus was resistant to those antibiotics. Antibiotic therapy can reduce differences in nasal microbiome (13). Although the sinus could be infected by more bacteria other than $S$. aureus, it is thought that the use of antibiotic effected to those bacteria and antibiotic resistant $S$. aureus remained, or solely infected by $S$. aureus. Unlike streptococcal infection, the isolate was resistant to penicillin and gentamicin, which had been previously used by the patient. S. aureus has the ability to produce biofilms to protect bacteria from host defenses and function as a barrier to some antibiotics (14). The icaA and icaD genes, which are the most widely researched genes, are responsible for the synthesis of biofilms by staphylococci (1). The biofilm formation ability of the isolates was determined molecularly using icaD gene detection by PCR (Fig. 4). Biofilm formation is one of the complex mechanisms of $S$. aureus (12). The isolates were determined to be multi-drug resistant $S$. aureus and not susceptible to penicillin, ampicillin, ceftiofur, and gentamicin (Table 1), which are regarded as routinely used drugs in the treatment of bacterial infections in equine practice (5). Multi-drug resistance of the isolated S. aureus might be acquired by frequent usage of these antibiotics in equine practice and the biofilm-producing characteristic of the isolate. Antimicrobial-associated diarrhea (AAD) is one of the main adverse effects of antimicrobial therapy in horses (2). It can result in longer hospitalization, additional expense for treatment, and especially fatality (2). C. butyricum is a probiotic that has been used in several gastrointestinal diseases in human medicine and has been clinically used in AAD (22). Since the patient had been administered prolonged antibiotic therapy for over 5 weeks, the status of feces and appetite were carefully checked on a daily basis, and the probiotics were supplemented to lower the risk of AAD. Fortunately, the patient did not develop AAD during antibiotic therapy.

Fast culture and sensitivity testing and the use of appropriate antibiotics are important for effective treatment of sinusitis (11). In this case, ineffective antibiotics were used initially, without bacterial isolation and antibiotic susceptibility testing. Delayed or ineffective treatment of primary sinusitis can lead to progression to chronic osteitis, advanced necrosis and destruction of soft tissue and bone, and inspissated abscesses (9). Such cases require more aggressive surgical treatments, such as a bone flap (9). Therefore, early bacterial isolation and antibiotic susceptibility testing and appropriate antibiotic therapy for bacterial sinusitis in horses should be initiated.

\section{Acknowledgements}

This study was supported by the Korea Racing Authority. The authors thank the KRA equine hospital staffs, Jihoon Jeong, Minhyun Lee, Kiyoung Jang, Youngjong Kim, Jeongwoo Choi, and Heonmin Park.

\section{Conflicts of Interest}

The authors have no conflicting interests.

\section{References}

\footnotetext{
1. Azmi K, Qrei W, Abdeen Z. Screening of genes encoding adhesion factors and biofilm production in methicillin resistant strains of Staphylococcus aureus isolated from Palestinian patients. BMC Genomics 2019; 20: 578.

2. Barr BS, Waldridge BM, Morresey PR, Reed SM, Clark C, Belgrave R, et al. Antimicrobial-associated diarrhoea in three equine referral practices. Equine Vet J 2013; 45: 154-158.
} 
3. Beste KJ, Lawhon SD, Chamoun-Emanuelli AM, Duff AH, Coleman $M C$, Griffin CE, et al. Culture-independent and dependent evaluation of the equine paranasal sinus microbiota in health and disease. Equine Vet J 2020; 52: 455-463.

4. Bladon B, Munroe G. Paranasal sinuses. In: Munroe G, Weese J, editors. Equine clinical medicine, surgery, and reproduction. Boca Raton: Taylor \& Francis Group. 2011: 395-400.

5. Choi SK, Hwang JY, Park CS, Cho GJ. Frequencies and antimicrobial susceptibility of methicillin-resistant Staphylococcus aureus (MRSA) isolated from horses in South Korea. Open Agric J 2018; 12: 148-155.

6. Choi SK, Park YS, Cho KH, Cho GJ. RAPD analysis and antimicrobial susceptibility of Streptococcus equi subsp. zooepidemicus isolated from thoroughbred horses. J Life Sci 2010; 20: 649-654.

7. Davis E. Disorders of the respiratory system. In: Reed SM, Bayly WM, Sellon DC, editors. Equine internal medicine. 4th ed. St. Loui: W.B. Saunders. 2018: 323-324.

8. Dixon PM, O'Leary JM. A review of equine paranasal sinusitis: medical and surgical treatments. Equine Vet Educ 2012; 24: 143158.

9. Freeman DE. Sinus disease. Vet Clin North Am Equine Pract 2003; 19: 209-243, viii.

10. Galvin N. Conditions of the respiratory tract. In: McAuliffe S, editor. Knottenbelt and Pascoe's color atlas of diseases and disorders of the horse. 2nd ed. Edinburgh: W.B. Saunders. 2014: 142-143.

11. Gordon DL, Radtke CL. Treatment of chronic sinusitis in a horse with systemic and intra-sinus antimicrobials. Can Vet J 2017; 58: 289-292.

12. Guo Y, Song G, Sun M, Wang J, Wang Y. Prevalence and therapies of antibiotic-resistance in Staphylococcus aureus. Front Cell Infect Microbiol 2020;10:107.

13. Kim YS, Han D, Mo JH, Kim YM, Kim DW, Choi HG, et al. Antibiotic-dependent relationships between the nasal microbiome and secreted proteome in nasal polyps. Allergy Asthma Immunol Res 2021; 13: 589-608.

14. Kot B, Sytykiewicz H, Sprawka I. Expression of the biofilm-associated genes in methicillin-resistant Staphylococcus aureus in biofilm and planktonic conditions. Int J Mol Sci 2018; 19: 3487.

15. Lubbers BV, Diaz-Campos DV, Schwarz S, Bowden R, Burbick CR,
Fajt VR, et al. Performance standards for antimicrobial disk and dilution susceptibility tests for bacteria isolated from animals. Wayne: Clinical \& Laboratory Standards Institute. 2020: 48-72.

16. Magiorakos AP, Srinivasan A, Carey RB, Carmeli Y, Falagas ME, Giske CG, et al. Multidrug-resistant, extensively drug-resistant and pandrug-resistant bacteria: an international expert proposal for interim standard definitions for acquired resistance. Clin Microbio Infect 2012; 18: 268-281.

17. Malo A, Cluzel C, Labrecque O, Beauchamp G, Lavoie JP, Leclere M. Evolution of in vitro antimicrobial resistance in an equine hospital over 3 decades. Can Vet J 2016; 57: 747-751.

18. Nickels F. Nasal passages and paranasal sinuses. In: Auer JA, Stick JA, editors. Equine surgery. 4th ed. St. Louis: Saunders Elsevier. 2012: 563-565.

19. Pascoe J. Sinusitis. In: Smith B, editor. Large animal internal medicine. 5th ed. St. Louis: Mosby Elsevier. 2015: 559-561.

20. Petti CA, Brandt ME, Church DL, Emler S, Simmon K, Zelazny AM. CLSI guideline MM18-A. Interpretive criteria for identification of bacteria and fungi by targeted DNA sequencing. Wayne: Clinical \& Laboratory Standards Institute. 2018: 25-26.

21. Schumacher J, Honnas C, Smith B. Paranasal sinusitis complicated by inspissated exudate in the ventral conchal sinus. Vet Surg 1987; 16: 373-377.

22. Sun YY, Li M, Li YY, Li LX, Zhai WZ, Wang P, et al. The effect of Clostridium butyricum on symptoms and fecal microbiota in diarrhea-dominant irritable bowel syndrome: a randomized, double-blind, placebo-controlled trial. Sci Rep 2018; 8: 2964.

23. Sweeney CR, Timoney JF, Newton JR, Hines MT. Streptococcus equi infections in horses: guidelines for treatment, control, and prevention of strangles. J Vet Intern Med 2005; 19: 123-134.

24. Tremaine WH, Dixon PM. A long-term study of 277 cases of equine sinonasal disease. Part 1: details of horses, historical, clinical and ancillary diagnostic findings. Equine Vet J 2001; 33: 274 282.

25. Witte T. Diseases of the nasal cavity and paranasal sinuses. In: Sprayberry K, Robinson NE, editors. Robinson's current therapy in equine medicine. 7th ed. St. Louis: Saunders Elsevier. 2015: 216217. 